\title{
Depression in myasthenia gravis: a heterogeneous and intriguing entity
}

\author{
Gavrilov, Yury V ; Alekseeva, Tatjana M ; Kreis, Olga A ; Valko, Philipp O ; Weber, Konrad P ; Valko,
} Yulia

\begin{abstract}
BACKGROUND Depressive symptoms in myasthenia gravis (MG) are common, may mimic other disease features, and contribute to misdiagnosis and diagnostic delay. Nevertheless, the clinical determinants of depressive symptoms in MG remain poorly studied, in particular their overlap with fatigue. Moreover, studies in MG have rarely looked at distinct depression phenotypes. METHODS In 68 consecutive MG patients, we ascertained cognitive-affective and somatic depression with the Beck Depression Inventory (BDI), and also assessed age at disease onset, education, marital state, work ability, sleepiness, fatigue, and treatment modalities. Disease severity was graded according to the Myasthenia Gravis Foundation of America (MGFA) classification. RESULTS The prevalence of moderate-severe depression was $20.5 \%$. While depression and fatigue showed large overlap ( $\mathrm{n}=37,54.4 \%$ ), only fatigue increased with disease severity, while BDI scores did not. Thymectomy was independently associated with lower BDI scores, but had no impact on fatigue. Total BDI scores were similar in patients with predominantly cognitive-affective and with predominantly somatic depression. However, ESS correlated only with cognitive-affective BDI, and younger age was independently associated with cognitive-affective BDI. Conversely, female sex and thymectomy were independently associated with somatic BDI. CONCLUSIONS Depression and fatigue are highly prevalent and largely overlapping comorbidities in MG, but only fatigue increased with disease severity, and only depression was milder in thymectomized patients. Comparative use of BDI subscales in MG reveals distinct depression phenotypes with distinct correlations to other disease features.
\end{abstract}

DOI: https://doi.org/10.1007/s00415-020-09767-7

Posted at the Zurich Open Repository and Archive, University of Zurich

ZORA URL: https://doi.org/10.5167/uzh-192434

Journal Article

Accepted Version

Originally published at:

Gavrilov, Yury V; Alekseeva, Tatjana M; Kreis, Olga A; Valko, Philipp O; Weber, Konrad P; Valko, Yulia (2020). Depression in myasthenia gravis: a heterogeneous and intriguing entity. Journal of Neurology, 267(6):1802-1811.

DOI: https://doi.org/10.1007/s00415-020-09767-7 


\title{
Depression in myasthenia gravis: a heterogeneous and intriguing entity
}

\author{
Yury V. Gavrilov ${ }^{1} \cdot$ Tatjana M. Alekseeva $^{2} \cdot$ Olga A. Kreis $^{2,3} \cdot$ Philipp O. Valko $^{4} \cdot$ Konrad P. Weber $^{4,5} \cdot$ Yulia Valko $^{4,5}$
}

Received: 20 December 2019 / Revised: 15 February 2020 / Accepted: 18 February 2020 / Published online: 5 March 2020

(c) Springer-Verlag GmbH Germany, part of Springer Nature 2020

\begin{abstract}
Background Depressive symptoms in myasthenia gravis (MG) are common, may mimic other disease features, and contribute to misdiagnosis and diagnostic delay. Nevertheless, the clinical determinants of depressive symptoms in MG remain poorly studied, in particular their overlap with fatigue. Moreover, studies in MG have rarely looked at distinct depression phenotypes. Methods In 68 consecutive MG patients, we ascertained cognitive-affective and somatic depression with the Beck Depression Inventory (BDI), and also assessed age at disease onset, education, marital state, work ability, sleepiness, fatigue, and treatment modalities. Disease severity was graded according to the Myasthenia Gravis Foundation of America (MGFA) classification.

Results The prevalence of moderate-severe depression was $20.5 \%$. While depression and fatigue showed large overlap $(n=37,54.4 \%)$, only fatigue increased with disease severity, while BDI scores did not. Thymectomy was independently associated with lower BDI scores, but had no impact on fatigue. Total BDI scores were similar in patients with predominantly cognitive-affective and with predominantly somatic depression. However, ESS correlated only with cognitive-affective BDI, and younger age was independently associated with cognitive-affective BDI. Conversely, female sex and thymectomy were independently associated with somatic BDI.

Conclusions Depression and fatigue are highly prevalent and largely overlapping comorbidities in MG, but only fatigue increased with disease severity, and only depression was milder in thymectomized patients. Comparative use of BDI subscales in MG reveals distinct depression phenotypes with distinct correlations to other disease features.
\end{abstract}

Keywords Myasthenia gravis · Depression · Beck Depression Inventory $\cdot$ Fatigue $\cdot$ Thymectomy

\section{Introduction}

Yulia Valko

Yulia.Valko@usz.ch

1 Department of General Pathology and Pathological Physiology, Institute of Experimental Medicine, 197376 St. Petersburg, Russia

2 Department of Neurology and Psychiatry, Almazov National Medical Research Centre, 197341 St. Petersburg, Russia

3 Department of Neurology, North-Western State Medical University, 191015 St. Petersburg, Russia

4 Department of Neurology, University Hospital Zurich, University of Zurich, 8091 Zurich, Switzerland

5 Department of Ophthalmology, University Hospital Zurich, University of Zurich, 8091 Zurich, Switzerland
Myasthenia gravis (MG) is a chronic autoimmune disease caused by antibodies against different epitopes of the neuromuscular junction [1]. The immune-mediated attack leads to a defective neuromuscular transmission, with patients experiencing a fluctuating, exercise-dependent, and usually reversible weakness in skeletal muscles. When recognizing the characteristic manifestation, clinicians can confirm the diagnosis of MG with the aid of various electrophysiological, immunological and pharmacological tests [2, 3]. It appears, however, that the correct diagnosis is missed in up to $46 \%$ of MG patients during the 1 st year of disease manifestation [4-6].

Depressive symptoms are a key confounder in MG, accounting for $20 \%$ of initial misdiagnoses [7]. Facial weakness and ptosis generally convey an impression of apathy and depressed mood, also in the absence of such 
feelings [8]. Moreover, the characteristic fluctuation of myasthenic symptoms can mislead to the assumption of a psychiatric origin. A simultaneous onset of MG and concurrent depressive symptoms adds to the risk of diagnostic confusion. Several groups have highlighted the potentially fatal consequences of a diagnostic delay, when MG had been mistaken for a psychiatric diagnosis [9-12].

Conversely, delayed recognition of depressive symptoms in MG patients may negatively impact the course of MG, and successful psychotherapy can lead to improvement of myasthenic symptoms [4, 13]. In an early study, psychiatric and emotional disturbances were associated with a twofold increased mortality in MG [14].

Psychopathological abnormalities have been reported to occur in up to $59 \%$ of MG patients, with depression being the most common disorder [15]. In a recent Danish study, suicide risk appeared to be significantly increased in MG compared to the general population [16]. However, despite growing awareness on the frequent and intriguing role of depressive symptoms in MG, they remain underdiagnosed and poorly studied, and some issues have not yet been addressed. First, depressive symptoms and fatigue are frequent and overlapping comorbidities in many neurological disorders, but this complex relationship has not yet been elucidated in MG. MG-related fatigue itself has only recently attracted more attention [17]. Moreover, the subjective perception of fatigue-a feeling of exhaustion, tiredness and loss of energy -is often confused with the objective clinical sign of myasthenic muscle fatigability [18], but represents also a prominent and defining component in the diagnosis of depression. For similar reasons, hence, both depressive symptoms and fatigue may pose a diagnostic challenge in MG. Second, both fatigue and depression are heterogeneous symptoms. We have previously shown that fatigue in MG was mainly physical, whereas cognitive fatigue levels did not differ between MG patients and controls [18]. Likewise, Paul et al. first emphasized the need to assess the different dimensions of depression in MG, after demonstrating that only the vegetative, but not the mood or evaluative subscales of the Chicago Multiscale Depression Inventory, differed between MG patients and controls [19]. The Beck Depression Inventory (BDI) is likely the most frequently used selfrating scale for measuring depression in both psychiatric and neurological samples, including also MG [20-24], but the cognitive-affective and somatic BDI subscales have not yet been separately examined in MG cohorts.

In the present study, we therefore aimed at assessing the prevalence and predictors of depressive symptoms in a well-characterized sample of consecutive MG patients, paying particular attention to the complex relationship with fatigue and to the heterogeneous entity of depression itself.

\section{Methods}

The present study is based on prospectively collected data of 73 consecutive MG patients seen between June 2014 and December 2017 at the Department of Neurology and Psychiatry of the Almazov National Medical Research Centre, St. Petersburg, Russia. The Ethics Committee of the Saint Petersburg State University approved the study protocol (No. 44-2012), and written informed consent for study participation was obtained from all participants. Using the same data set, we have previously reported on other MG-related issues, including fatigue [18], and the role of autoimmune comorbidity [24]. We conducted the study in accordance with the Declaration of Helsinki.

\section{Study population}

Demographic and clinical characteristics of the included MG patients comprised age, sex, body mass index (BMI), marital status (single, married or divorced) and working ability. Education status was defined as the highest degree attained (primary school, college school, university). The diagnosis of MG was based on a typical clinical manifestation and confirmed by the positivity of at least one of the following [25, 26]: demonstration of a pathognomonic decrement in repetitive nerve stimulation, a positive response of the muscular weakness to the edrophonium test and/or to the subsequent pharmacological treatment, and detection of MG-specific antibodies such as anti-acetylcholine receptor (AChR) antibodies or striational antibodies, i.e. antibodies directed against intracellular proteins of striated muscles (titin, actin, myosin, ryanodine receptors) [27]. All patients with positive striational antibodies had also positive AChR antibodies. In a single patient with positive striational antibodies, AChR antibody profile was not determined. In addition, we ascertained the following disease variables in all patients: age at disease onset, disease duration at study conduction, disease disability as graded by the Myasthenia Gravis Foundation of America (MGFA) classification, and treatment type, including pharmacological therapy with corticosteroids and/or immunosuppressive agents, as well as whether or not the patients had been subjected to thymectomy or plasmapheresis. Due to incomplete data, we excluded five patients, i.e. the final study sample comprised $68 \mathrm{MG}$ patients. Only two patients were under antidepressive pharmacotherapy at the time of study assessment.

\section{Self-administered questionnaires}

We assessed the frequency and severity of depressive symptoms by administering the validated Russian version of the 
Beck Depression Inventory (BDI), one of the most widely used self-rating scales to measure depression [28, 29]. The BDI contains 21 items with statements referring to the last week and the patient has to rate them on a score of $0-3$. The highest possible total BDI score is 63 . Based on the total BDI score, we graded the severity of depressive symptoms as mild (10-18), moderate (19-29), or severe $(\geq 30)$. The BDI is commonly divided into a cognitive-affective domain (items 1-13), and a somatic domain (items 14-21). Depending on the subscale with the higher rating, we dichotomized the patients into a cognitive-affective predominance and a somatic predominance group. The scale has been validated for many psychiatric and neurological samples [30-32], but not yet for MG. In the present sample, Cronbach's alpha of the BDI was 0.80 , indicating satisfactory internal consistency.

To measure the patients' severity of fatigue and its impact on various daily domains, we used the Fatigue Severity Scale (FSS) and the Fatigue Impact Scale (FIS), respectively. The Fatigue Severity Scale (FSS) is a nine-item questionnaire on the degree of mainly physical fatigue experienced during the last week [33]. For each item, patients rate their agreement/ disagreement with the statements on a seven-point Likert scale. The final FSS score reflects the average score of the nine items, with 1.0 and 7.0 representing minimal and maximal fatigue severity, respectively. We used a cutoff of $\geq 4.0$ for clinically relevant fatigue, as used in most validation studies [34]. The FIS is a 40-item self-report scale evaluating the consequences of fatigue on various domains of daily living [35]. Ten items relate to the impact of fatigue on cognitive aspects, ten items on physical aspects, and 20 items on psychosocial aspects. For each item, patients have to indicate how often they have experienced the described situation during the last month $(0=$ never, $1=$ rarely, $2=$ sometimes, $3=$ often, $4=$ always). The sum of the three subscales ranges from 0 to 40 (physical and cognitive FIS) and 0 to 80 (psychosocial FIS). The Russian versions of both fatigue scales have been validated by previous work $[18,36]$. Eventually, we used the Epworth Sleepiness Scale (ESS) to measure daytime sleepiness [37].

\section{Statistical analysis}

We used SPSS for statistical analyses (version 25; IBM, Armonk, New York, NY, USA). Group data were described by means and standard deviations. We used Student's $t$ test to compare normally distributed data between two groups, and applied Chi-square test in case of nominal data. Pearson's $r$ coefficient was applied for correlation analyses. To identify independent associates of higher BDI scores as well as the cognitive-affective and somatic BDI subscales, we employed stepwise multiple regression analyses, including the following potential predictor variables in the model: age, sex, age at MG onset, BMI, education status, marital status, MGFA, FSS, FIS physical, FIS cognitive, FIS psychosocial, ESS, and thymectomy. Differences with $p$ values $<0.05$ were considered statistically significant.

\section{Results}

\section{Prevalence and determinants of depression in MG}

Mean BDI was $12.9 \pm 7.3$ (cognitive-affective BDI 7.2 \pm 4.8 , somatic BDI $5.7 \pm 3.7$ ), with 47 MG patients $(64.7 \%$ ) having BDI scores $\geq 10$. Depression was mild in 30 patients $(44.1 \%)$, moderate in 12 patients $(17.6 \%)$, and severe in 2 patients (2.9\%). Depressed and non-depressed MG patients did not differ with respect to age, sex, BMI, or disease severity and duration (Table 1). Fatigue prevalence was higher in depressed than non-depressed patients $(84.1 \%$ vs. $45.8 \%$, $p=0.002$ ), including significantly higher scores in all FIS subscales. Depressed patients also had higher ESS scores. The psychosocial FIS subscale appeared to exhibit the strongest difference between depressed and non-depressed MG patients. Multiple regression analysis confirmed psychosocial FIS as independent predictor of higher BDI scores ( $\beta=0.626, t$ value $=6.820, p<0.001)$.

There was a large overlap between depression and fatigue $(n=37)$ (Fig. 1a), i.e. the concurrent prevalence of depression and fatigue was $54.4 \%$. BDI and FSS scores showed a strong correlation $(r=0.492, p<0.001)$ (Fig. 1b). Only a minority had depression without fatigue $(n=7)$. MG patients with isolated depression had significantly milder disease severity than those with concurrent depression and fatigue or isolated fatigue (Fig. 1c). Fatigue increased with disease severity, whereas depressive symptoms were independent of MGFA disease stage (Fig. 2). Specifically, there was no association of MGFA category with either the cognitiveaffective BDI subscale $(r=-0.035, p=0.78)$ nor with the somatic BDI subscale $(r=0.170, p=0.17)$.

\section{Impact of thymectomy on depression in MG}

Thymectomy was performed in 18 patients $(26.5 \%)$, with a mean latency from disease onset of $1.5 \pm 0.9$ years (range $1-4$ years), and $9.0 \pm 7.5$ years before study completion. MG patients with a history of thymectomy had a lower prevalence of depression than those without thymectomy (15.9\% vs. $45.8 \%, p=0.01)$ and their BDI scores were significantly lower $(9.1 \pm 5.8$ vs. $14.3 \pm 7.3, p=0.007)$ (Table 2). MG patients with and without thymectomy did not differ with respect to age, MGFA category, current treatment type, ESS, or FSS and FIS scores. MG patients with a history of thymectomy, however, were younger at disease onset ( $30 \pm 12.8$ years vs. $40.8 \pm 17.3$ years, $p=0.02)$, and their 
Table 1 Comparison of myasthenia gravis (MG) patients with and without depression

\begin{tabular}{|c|c|c|c|}
\hline & $\begin{array}{l}\text { Depression } \\
(n=44)\end{array}$ & $\begin{array}{l}\text { No depression } \\
(n=24)\end{array}$ & $p$ \\
\hline Age (years) & $45.6 \pm 15.5$ & $45.4 \pm 14.8$ & 0.97 \\
\hline Female sex & $26(59.1)$ & $14(58.3)$ & 1.00 \\
\hline Body mass index & $28.5 \pm 7.7$ & $27.2 \pm 6.1$ & 0.47 \\
\hline Duration of MG (years) & $7.6 \pm 7.7$ & $7.3 \pm 7.4$ & 0.85 \\
\hline Age at MG onset (years) & $37.9 \pm 16.8$ & $38.1 \pm 17.3$ & 0.97 \\
\hline Epworth Sleepiness Scale (ESS) & $7.6 \pm 4.2$ & $5.2 \pm 2.2$ & 0.01 \\
\hline Excessive daytime sleepiness (ESS $\geq 11$ ) & $10(22.7)$ & $1(4.2)$ & 0.04 \\
\hline Unable to work & $19(43.2)$ & $9(37.5)$ & 0.80 \\
\hline Marital state & & & 0.37 \\
\hline Single & $14(31.8)$ & $4(16.7)$ & \\
\hline Married & $25(56.8)$ & $17(70.8)$ & \\
\hline Divorced & $4(9.1)$ & $3(12.5)$ & \\
\hline Education & & & 0.58 \\
\hline Primary school degree & $16(36.4)$ & $10(41.7)$ & \\
\hline College school degree & $5(11.4)$ & $1(4.2)$ & \\
\hline University degree & $21(47.7)$ & $13(54.2)$ & \\
\hline Myasthenia Gravis Foundation of America & & & 0.83 \\
\hline I & $6(13.6)$ & $5(20.8)$ & \\
\hline IIa & $10(22.7)$ & $7(29.2)$ & \\
\hline $\mathrm{IIb}$ & $6(13.6)$ & $4(16.7)$ & \\
\hline IIIa & $16(36.4)$ & $6(25)$ & \\
\hline IIIb & $5(11.4)$ & $2(8.3)$ & \\
\hline IVa & $1(2.3)$ & $0(0)$ & \\
\hline \multicolumn{4}{|l|}{ Current therapy } \\
\hline Steroids & $20(45.5)$ & $13(54.2)$ & 0.61 \\
\hline Current dose of prednisolone (mg/days) & $10.4 \pm 18.7$ & $11.2 \pm 18.1$ & 0.86 \\
\hline Immunosuppressants $^{\mathrm{a}}$ & $11(25)$ & $6(25)$ & 1.00 \\
\hline Plasmapheresis & $14(31.8)$ & $9(37.5)$ & 0.36 \\
\hline Thymectomy & $7(15.9)$ & $11(45.8)$ & 0.01 \\
\hline Fatigue Severity Scale (FSS) & $5.4 \pm 1.6$ & $3.7 \pm 1.9$ & $<0.001$ \\
\hline Fatigue frequency $(\mathrm{FSS} \geq 4.0)$ & $37(84.1)$ & $11(45.8)$ & 0.002 \\
\hline Fatigue Impact Scale, cognitive & $14.1 \pm 8.4$ & $8.5 \pm 7.0$ & 0.007 \\
\hline Fatigue Impact Scale, physical & $26 \pm 8.7$ & $16.8 \pm 7.9$ & $<0.001$ \\
\hline Fatigue Impact Scale, psychosocial & $40 \pm 14.3$ & $22 \pm 13.9$ & $<0.001$ \\
\hline
\end{tabular}

Bold values indicate statistical significance

a azathioprine $n=15$, cyclophosphamide $n=1$, vincristine $n=1$ disease duration at study completion was, hence, longer than in MG patients without thymectomy $(10.5 \pm 7.4$ years vs. $6.4 \pm 7.4$ years, $p=0.05$ ). Multiple linear regression analysis confirmed thymectomy as independent associate of lower BDI scores $(\beta=-0.219, t$ value $=-2.389, p<0.001)$.

\section{Cognitive-affective vs. somatic depression in MG}

Among the entire sample, $25 \mathrm{MG}$ patients had higher scores in the cognitive-affective subscale of the BDI, while $43 \mathrm{MG}$ patients had higher scores in the somatic subscale. Among the 44 depressed MG patients, 12 had predominantly cognitive-affective depression, while 32 had predominantly somatic depression (Table 3). Whether MG patients suffered predominantly from cognitive-affective or somatic depression did not affect the total BDI score (18.2 \pm 6.5 vs. $16.5 \pm 5.5, p=0.40)$. Patients with predominantly cognitive-affective depression were younger than those with predominantly somatic depression and had lower scores in all fatigue scales except in the FIS cognitive. The FSS showed a stronger correlation with the somatic BDI subscale $(r=0.588, p<0.001)$ than with the cognitive-affective BDI subscale $(r=0.293, p=0.02)$. Conversely, the ESS correlated only with the cognitive-affective $(r=0.26, p=0.03)$ but 


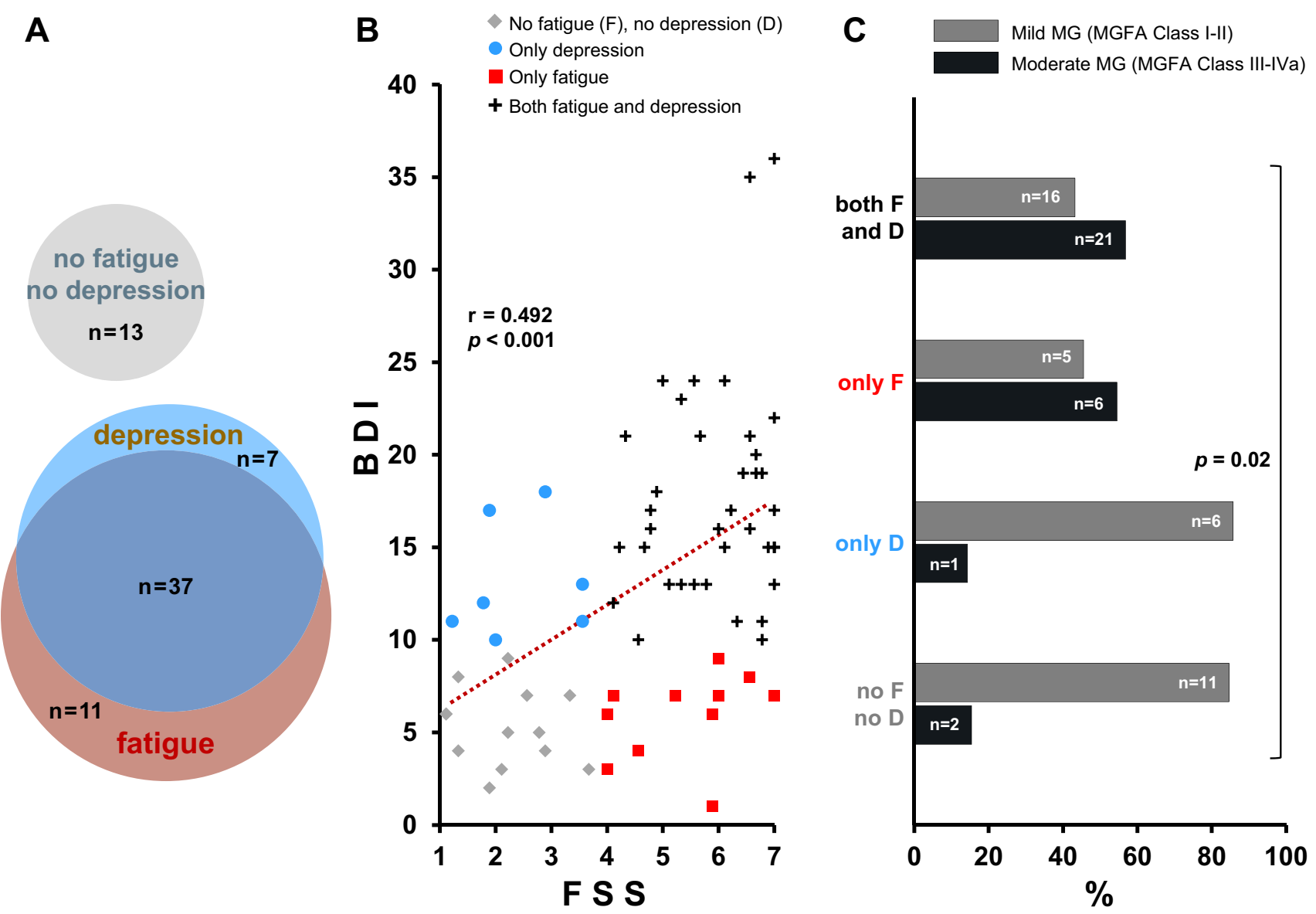

Fig. 1 Fatigue and depression largely overlap in patients with myasthenia gravis (MG), with a minority having only one of the two symptoms (a). BDI and FSS scores exhibit a strong correlation (b), but depression and fatigue are differentially associated with disease severity (c). Moderate disease severity was more common in MG patients manifesting both fatigue and depression (56.8\%) or isolated fatigue $(54.5 \%)$ than in patients with isolated depression $(14.3 \%)$ or without fatigue/depression (15.4\%)
Fig. 2 The scores of the Fatigue Severity Scale (FSS)—but not of the Beck Depression Inventory (BDI) - increased with disease severity, as measured by the Myasthenia Gravis Foundation of America (MGFA) classification
A

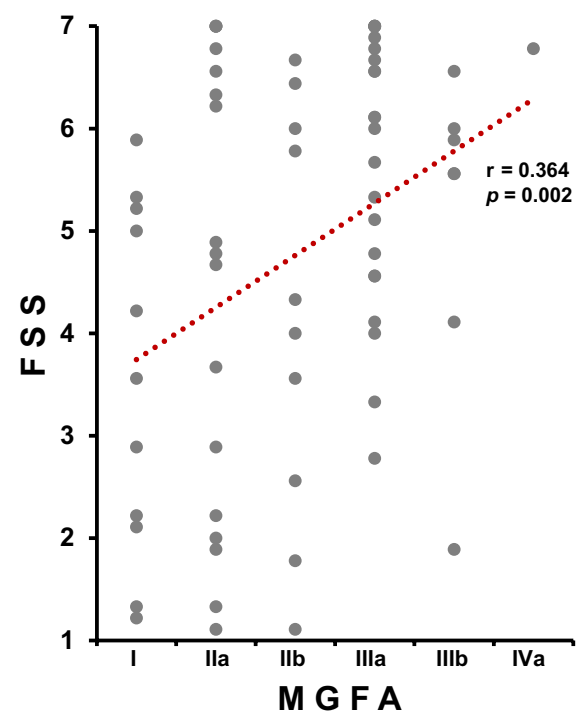

B

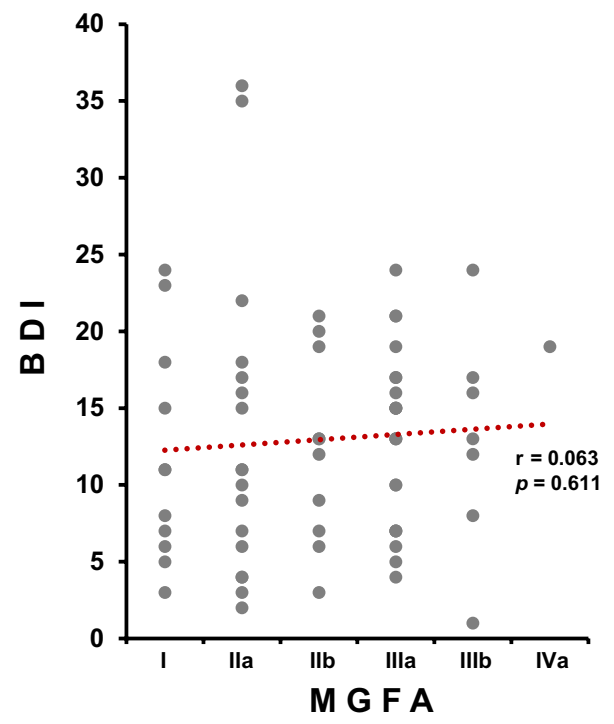


Table 2 Comparison of myasthenia gravis (MG) patients with and without thymectomy

\begin{tabular}{|c|c|c|c|}
\hline & $\begin{array}{l}\text { Thymectomy } \\
(n=18)\end{array}$ & $\begin{array}{l}\text { No thymectomy } \\
(n=50)\end{array}$ & $p$ \\
\hline Age (years) & $40.5 \pm 12.6$ & $47.3 \pm 15.7$ & 0.10 \\
\hline Female sex & $13(72.2)$ & $27(54)$ & 0.27 \\
\hline Body mass index & $26.8 \pm 5.0$ & $28.5 \pm 7.8$ & 0.40 \\
\hline Duration of MG (years) & $10.5 \pm 7.4$ & $6.4 \pm 7.4$ & 0.05 \\
\hline Age at MG onset (years) & $30 \pm 12.8$ & $40.8 \pm 17.3$ & 0.02 \\
\hline Epworth Sleepiness Scale & $6.4 \pm 4.2$ & $6.9 \pm 3.7$ & 0.68 \\
\hline Unable to work & $6(33.3)$ & $22(44)$ & 0.58 \\
\hline Marital state & & & 0.57 \\
\hline Single & $4(22.2)$ & $14(28)$ & \\
\hline Married & $11(61.1)$ & $31(62)$ & \\
\hline Divorced & $3(16.7)$ & $4(8)$ & \\
\hline Education & & & 0.32 \\
\hline Primary school degree & $5(27.8)$ & $21(42)$ & \\
\hline College school degree & $1(5.6)$ & $5(10)$ & \\
\hline University degree & $12(66.7)$ & $22(44)$ & \\
\hline Myasthenia Gravis Foundation of America & & & 0.58 \\
\hline I & $2(11.1)$ & $9(18)$ & \\
\hline IIa & $6(33.3)$ & $11(22)$ & \\
\hline Iib & $1(5.6)$ & $9(18)$ & \\
\hline IIIa & $6(33.3)$ & $16(32)$ & \\
\hline IIIb & $3(16.7)$ & $4(8)$ & \\
\hline IVa & $0(0)$ & $1(2)$ & \\
\hline \multicolumn{4}{|l|}{ Therapy } \\
\hline Current treatment with steroids & $11(61.1)$ & $22(44)$ & 0.28 \\
\hline Current dose of oral steroids & $9.1 \pm 17.3$ & $11.3 \pm 18.9$ & 0.67 \\
\hline Duration of immunosuppressive therapy (years) & $0.7 \pm 1.1$ & $0.3 \pm 1.1$ & 0.18 \\
\hline Beck Depression Inventory (BDI) & $9.1 \pm 5.8$ & $14.3 \pm 7.3$ & 0.007 \\
\hline Moderate-severe depression & $2(11 \%)$ & $12(24 \%)$ & 0.21 \\
\hline Fatigue Severity Scale (FSS) & $4.3 \pm 1.7$ & $4.9 \pm 1.9$ & 0.28 \\
\hline Fatigue frequency $(\mathrm{FSS} \geq 4.0)$ & $11(61.1)$ & $37(74)$ & 0.37 \\
\hline Fatigue Impact Scale, cognitive & $11.4 \pm 8.6$ & $12.3 \pm 8.3$ & 0.69 \\
\hline Fatigue Impact Scale, physical & $21.2 \pm 9.5$ & $23.3 \pm 9.5$ & 0.43 \\
\hline Fatigue Impact Scale, psychosocial & $29.6 \pm 17.8$ & $35.1 \pm 16$ & 0.23 \\
\hline
\end{tabular}

Bold values indicate statistical significance not with the somatic BDI subscale $(r=0.17, p=0.16)$. Multiple linear regression models revealed different independent associates of the two BDI subscales (Table 4).

\section{Discussion}

Our study confirms that depressive symptoms are highly prevalent in MG. The $20.5 \%$ frequency of moderate-severe depression in our cohort is comparable to other BDI-based assessments in MG, with frequencies ranging from 13.6 to $33 \%[21-23,38]$. The devastating and unpredictable nature of the disease, social disadvantages, and adverse effects of chronic corticosteroid treatment may all account for the high frequency of depressive symptoms in MG [39]. In addition, the underlying immunopathology in MG may contribute to a higher susceptibility for depression, as suggested by the growing evidence linking depression to abnormalities in both the innate and adaptive immune systems [40-44]. In this regard, it is tempting to speculate that the lower frequency of depressive symptoms in thymectomized MG patients might be attributable in part to changes in the immune system.

In the present study, psychosocial fatigue appeared as the strongest independent associate of higher BDI scores. While the negative impact of depression on health-related quality of life in MG is well established [45-50], studies looking specifically at potential predictors of depressive symptoms 
Table 3 Comparison of myasthenia gravis patients with depression $(n=44)$, dichotomized according to the type of depression

\begin{tabular}{|c|c|c|c|}
\hline & $\begin{array}{l}\text { Predominantly } \\
\text { cognitive-affective } \\
(n=12)\end{array}$ & $\begin{array}{l}\text { Predominantly somatic } \\
(n=32)\end{array}$ & $p$ \\
\hline Age (years) & $35.3 \pm 13.9$ & $49.4 \pm 14.4$ & 0.005 \\
\hline Female sex & $4(33.3)$ & $22(68.8)$ & 0.05 \\
\hline Body mass index & $24.8 \pm 3.5$ & $29.9 \pm 8.4$ & 0.05 \\
\hline Duration of MG (years) & $7.8 \pm 7.0$ & $7.6 \pm 8.1$ & 0.94 \\
\hline Age at MG onset (years) & $27.5 \pm 16.4$ & $41.8 \pm 15.4$ & 0.01 \\
\hline Epworth Sleepiness Scale & $9.4 \pm 4.9$ & $7.0 \pm 3.8$ & 0.09 \\
\hline Unable to work & $4(33.3)$ & $15(46.9)$ & 0.51 \\
\hline Marital state & & & 0.20 \\
\hline Single & $6(50)$ & $8 / 31(25.8)$ & \\
\hline Married & $6(50)$ & $19 / 31(61.3)$ & \\
\hline Divorced & $0(0)$ & 4/31 (12.9) & \\
\hline Education & & & 0.18 \\
\hline Primary school degree & $3 / 11(27.3)$ & 13/31 (41.9) & \\
\hline College school degree & $3 / 11(27.3)$ & $2 / 31(6.5)$ & \\
\hline University degree & $5 / 11(45.5)$ & 16/31 (51.6) & \\
\hline Myasthenia Gravis Foundation of America & & & 0.07 \\
\hline I & $3(25)$ & $3(9.4)$ & \\
\hline IIa & $2(16.7)$ & $8(25)$ & \\
\hline $\mathrm{IIb}$ & $3(25)$ & $3(9.4)$ & \\
\hline IIIa & $1(8.3)$ & $15(46.9)$ & \\
\hline IIIb & $3(25)$ & $2(6.3)$ & \\
\hline IVa & $0(0)$ & $1(3.1)$ & \\
\hline \multicolumn{4}{|l|}{ Autoantibody profile } \\
\hline Acetylcholine receptor antibodies & $8 / 10(80)$ & $23 / 27(85.2)$ & 0.53 \\
\hline \multicolumn{4}{|l|}{ Therapy } \\
\hline Current treatment with steroids & $7(58.3)$ & $13(40.1)$ & 0.33 \\
\hline Thymectomy & $3(25)$ & $4(12.5)$ & 0.37 \\
\hline Beck Depression Inventory (BDI) & $18.2 \pm 6.5$ & $16.5 \pm 5.5$ & 0.40 \\
\hline Fatigue Severity Scale (FSS) & $4.4 \pm 1.8$ & $5.7 \pm 1.4$ & 0.02 \\
\hline Fatigue frequency $(\mathrm{FSS} \geq 4.0$ ) & $7(58.3)$ & $30(93.8)$ & 0.01 \\
\hline Fatigue Impact Scale, cognitive & $12.8 \pm 8.9$ & $14.5 \pm 8.3$ & 0.54 \\
\hline Fatigue Impact Scale, physical & $20.6 \pm 9.7$ & $28.0 \pm 7.5$ & 0.01 \\
\hline Fatigue Impact Scale, psychosocial & $32.8 \pm 13.8$ & $42.7 \pm 13.7$ & 0.04 \\
\hline
\end{tabular}

Bold values indicate statistical significance

\begin{tabular}{llccr}
\hline Dependent variable & Significant coefficients & Beta & $t$ value & \multicolumn{1}{c}{$p$} \\
\hline Cognitive-affective BDI subscale & FSS score & 0.340 & 2.893 & 0.005 \\
& Age & -0.252 & -2.141 & 0.036 \\
Somatic BDI subscale & FSS score & 0.476 & 5.149 & $<0.001$ \\
& Thymectomy & -0.353 & -3.856 & $<0.001$ \\
& Female sex & 0.287 & 3.089 & 0.003 \\
\hline
\end{tabular}

${ }^{\text {a }}$ The following additional coefficients were included in the model: marital status, education status, body mass index, duration of MG, MGFA stage, ESS score 
in MG are very rare. A multicenter study from Eastern Japan assessed 287 consecutive MG patients by means of the BDIII; they found a $13.6 \%$ prevalence, and identified dose of oral corticosteroids, negative treatment response during early disease stage, and disease severity as independent associates of moderate-severe depression [22]. Fatigue was not included as a variable in this study.

Depressive symptoms and fatigue showed a large overlap, but correlated differentially with other disease features. Specifically, severity of fatigue - but not of depressive symptoms-increased with higher MGFA stages. Several factors may account for the large overlap between depressive symptoms and fatigue in MG. First, fatigue can primarily represent a symptom of depression. Second, fatigue is primarily a symptom of MG and secondarily contributes to depression. Third, depressive symptoms and fatigue may share a common underlying aetiology. Fourth, depressive symptoms and fatigue arise independently and overlap merely by chance. Obviously, the observational character of the present study does not allow teasing apart from the various directions of causality between depressive symptoms and fatigue, even less when considering also the multidimensional nature of both symptoms.

It has been argued that the use of the BDI might overestimate the true prevalence of depressive symptoms in MG, because it contains several somatic items. Many reasons indicate, however, that the high frequency of depressive symptoms in MG should not be downplayed as measurement error caused by the somatic BDI items. (1) In our MG sample, BDI scores-including the somatic BDI subscaledid not correlate with disease severity, in line with other studies [23]. (2) While the item on sleep quality belongs to the somatic BDI subscale, severity of daytime sleepiness correlated only with the cognitive-affective BDI subscale, but not with the somatic subscale. (3) The BDI has been successfully validated in various neurological disorders with significant somatic disability, including ischemic stroke [31], Parkinson's disease [30], and multiple sclerosis [32]. (4) When assessing depressive symptoms in MG with different scales, the BDI-based prevalence of depression appeared to be lower $(40.5 \%)$ than that measured by the Hamilton Depression Scale (52.4\%) [23]. (5) In an earlier study, a psychiatrist evaluated $74 \mathrm{MG}$ patients using a semistructured interview along the Diagnostic and Statistical Manual of Mental Disorders (DSM-III), revealing a 51\% prevalence of psychiatric disturbances and a $22 \%$ prevalence of depression, the latter number closely matching the $20.5 \%$ frequency of moderate-severe depression in our cohort [51].

Finally, our findings highlight the importance of assessing the various components of depressive symptoms in MG, as indicated by the differential associations of the BDI subscales with other disease features. MG patients with a predominance in cognitive-affective depression differed in various ways from MG patients with predominantly somatic depression, yet both groups had similar total BDI scores. Specifically, predominance in cognitive-affective depression was associated with younger age and earlier disease onset. In addition, ESS scores correlated only with the cognitiveaffective but not with the somatic BDI subscale. Female sex, on the other hand, appeared as independent associate of higher somatic depression. This latter finding, together with similar scores in total BDI and cognitive-affective BDI subscale, is of particular interest with regard to the robust epidemiological and clinical evidence of a higher frequency of depression in women than in men [52]. It suggests that MG-related causes of depressive symptoms prevail over the social and psychological factors usually believed to be responsible for the female preponderance in depression.

Our study has several limitations. First, we did not include a control group, which would have allowed for better differentiation between MG-specific associations of depressive symptoms and more general characteristics of depressive symptoms. Second, the patient sample was rather small and some results warrant replication in a larger MG cohort. Third, our MG sample lacks patients with higher MGFA category and generalization of our findings is therefore restricted. The underrepresentation of severely affected MG patients may have contributed to the negative association between severity of depressive symptoms and MGFA scores. Nevertheless, the fairly homogeneous distribution of our patients over four distinct MGFA stages (I, IIa, IIb, IIIa), the high frequency of depressive symptoms in our cohort, and a similar observation by another group [Aysal] indicate that the lack of correlation between depressive symptoms and disease severity cannot be attributed merely to the fact that we have not included very severely affected patients. Finally, patient characteristics did not include other psychosocial factors potentially contributing to depressive symptoms, such as social support, social disadvantage, or financial burden.

In conclusion, we showed that depression in MG represents a highly prevalent, complex and multifaceted symptom, as indicated by the overlap with and differences from fatigue and by the distinct clinical associations of cognitiveaffective and somatic depression. Future studies may want to delineate the specific diagnostic challenges and differing therapeutic requirements imposed by the prevailing depression phenotype.

Acknowledgements This study was supported by the Swiss National Science Foundation [320030_166346] and the Uniscientia Stiftung, Vaduz, Liechtenstein.

\section{Compliance with ethical standards}

Conflicts of interest The authors declare that they have no conflict of interest. 


\section{References}

1. Gilhus NE, Verschuuren JJ (2015) Myasthenia gravis: subgroup classification and therapeutic strategies. Lancet Neurol 14(10):1023-1036

2. Gilhus NE (2016) Myasthenia gravis. N Engl J Med 375:2570-2581

3. Valko Y, Rosengren SM, Jung HH, Straumann D, Landau K, Weber KP (2016) Ocular vestibular evoked myogenic potentials as a test for myasthenia gravis. Neurology 86:660-668

4. Sneddon J (1980) (1980) Myasthenia gravis-the difficult diagnosis. Br J Psychiatry 136:92-93

5. Beghi E, Antozzi C, Batocchi AP, Cornelio F, Cosi V, Evoli A, Lombardi M, Mantegazza R, Monticelli ML, Piccolo G, Tonali P, Trevisan D, Zarrelli M (1991) Prognosis of myasthenia gravis: a multicenter follow-up study of 844 patients. J Neurol Sci 106:213-220

6. Kalb B, Matell G, Pirskanen R, Lambe M (2002) Epidemiology of myasthenia gravis: a population-based study in Stockholm, Sweden. Neuroepidemiology 21:221-225

7. Rohr W (1992) Myasthenia gravis in the frontier of psychiatric diagnosis. Psychiatr Prax 19:157-163

8. Lázaro E, Amayra I, López-Paz JF, Jometón A, Martín N, Caballero P, De Nicolás L, Hoffmann H, Kessler H, Ruiz B, Martínez O (2013) Facial affect recognition in myasthenia gravis. Span J Psychol 16:E52

9. Santy PA (1983) Undiagnosed myasthenia gravis in emergency psychiatric referrals. Ann Emerg Med 12:397-398

10. Shinkai K, Ohmori O, Ueda N, Nakamura J, Amano T, Tsuji S (2001) A case of myasthenia gravis preceded by major depression. J Neuropsychiatr Clin Neurosci 13:116-117

11. Rüegg SJ, Dirnhofer S, Buitrago-Tellez CH, Steck AJ, Marsch S (2007) Life-threatening myasthenia gravis masked by a psychiatric disorder. Swiss Arch Neurol Psychiatry 158:150-154

12. Okamoto N, Furusawa Y, Sakamoto K, Yamamoto T, Kondo Y, Nagafusa Y, Higuchi T (2010) Major depression: what caused the crisis? The Lancet $375: 346$

13. Kulaksizoglu IB (2007) Mood and anxiety disorders in patients with myasthenia gravis; aetiology, diagnosis and treatment. CNS Drugs 21:473-481

14. Meyer E (1966) Psychological disturbances in myasthenia gravis: a predictive study. Ann N Y Acad Sci 135:417-423

15. Doering S, Henze T, Schussler G (1993) Coping with myasthenia gravis and implications for psychotherapy. Arch Neurol 50:617-620

16. Eliasen A, Dalhoff KP, Horwitz H (2018) Neurological diseases and risk of suicide attempt: a case-control study. J Neurol 265:1303-1309

17. Tran C, Bril V, Katzberg HD, Barnett C (2018) Fatigue is a relevant outcome in patients with myasthenia gravis. Muscle Nerve 58:197-203

18. Alekseeva TM, Gavrilov YV, Kreis OA, Valko PO, Weber KP, Valko Y (2018) Fatigue in patients with myasthenia gravis. J Neurol 265:2312-2321

19. Paul RH, Cohen RA, Goldstein JM, Gilchrist JM (2000) Severity of mood, self-evaluative, and vegetative symptoms of depression in myasthenia gravis. J Neuropsychiatr Clin Neurosci 12:499-501

20. Glennerster A, Palace J, Warburton D, Oxbury S, NewsomDavis J (1996) Memory in myasthenia gravis: neuropsychological tests of central cholinergic function before and after effective immunologic treatment. Neurology 46:1138-1142

21. Fisher J, Parkinson K, Kothari MJ (2003) Self-reported depressive symptoms in myasthenia gravis. J Clin Neuromuscul Dis 4:105-108
22. Suzuki Y, Utsugisawa K, Suzuki S, Nagane Y, Masuda M, Kabasawa C, Shimizu Y, Utsumi H, Uchiyama S, Fujihara K, Suzuki N (2011) Factors associated with depressive state in patients with myasthenia gravis: a multicentre cross-sectional study. BMJ Open 1:e000313

23. Aysal F, Karamustafalioğlu O, Özçelik B, Yilmaz M, Karamustafalioğlu N, Yumrukçal H, Tankaya O (2013) The relationship of symptoms of anxiety and depression with disease severity and treatment modality in myasthenia gravis: a crosssectional study. Arch Neuropsychiatr 50:295-300

24. Alekseeva TM, Kreis OA, Gavrilov YV, Valko PO, Weber KP, Valko Y (2019) Impact of autoimmune comorbidity on fatigue, sleepiness and mood in myasthenia gravis. J Neurol 266:2027-2034

25. Alekseeva TM, Kosachev VD, Khalmurzina AN (2016) Clinical and immunological features and treatment of myasthenia gravis in the elderly (review). Neuromuscul Dis 6:10-16

26. Kosachev VD, Lobzin SV, Alekseeva TM, Kreis OA (2015) Clinical and neurological criteria for the diagnosis of myasthenia gravis. Herald of the Northwestern State Medical University named after I.I. Mechnikov 7:102-107

27. Meriggioli MN, Sanders DB (2012) Muscle autoantibodies in myasthenia gravis: beyond diagnosis? Expert Rev Clin Immunol 8:427-438

28. Beck AT, Ward CH, Mendelson M, Mock J, Erbaugh J (1961) An inventory for measuring depression. Arch Gen Psychiatry 4:561-571

29. Andriushchenko AV, Drobizhev MIu, Dobrovol'skiı̌ AV (2003) A comparative validation of the scale CES-D, BDI, and HADS(d) in diagnosis of depressive disorders in general practice. Zhurnal Nevrologii i Psikhiatrii imeni S.S Korsakova 103:11-18

30. Visser M, Leentjens AF, Marinus J, Stiggelbout AM, van Hilten JJ (2006) Reliability and validity of the Beck depression inventory in patients with Parkinson's disease. Mov Disord 21:668-672

31. Berg A, Lönnqvist J, Palomäki H, Kaste M (2009) Assessment of depression after stroke: a comparison of different screening instruments. Stroke 40:523-529

32. Sacco R, Santangelo G, Stamenova S, Bisecco A, Bonavita S, Lavorgna L, Trojano L, D'Ambrosio A, Tedeschi G, Gallo A (2016) Psychometric properties and validity of Beck Depression Inventory II in multiple sclerosis. Eur J Neurol 23:744-750

33. Krupp LB, LaRocca NG, Muir-Nash J, Steinberg AD (1989) The fatigue severity scale. Application to patients with multiple sclerosis and systemic lupus erythematosus. Arch Neurol 46:1121-1123

34. Valko PO, Bassetti CL, Bloch KE, Held U, Baumann CR (2008) Validation of the fatigue severity scale in a Swiss cohort. Sleep 31:1601-1607

35. Fisk JD, Ritvo PG, Ross L, Haase DA, Marrie TJ, Schlech WF (1994) Measuring the functional impact of fatigue: initial validation of the fatigue impact scale. Clin Infect Dis 18:S79-83

36. Gavrilov YV, Shkilnyuk GG, Valko PO, Stolyarov ID, Ivashkova EV, Ilves AG, Nikiforova IG, Shchelkova OY, Vasserman LI, Vais EE, Valko Y (2018) Validation of the Russian version of the fatigue impact scale and fatigue severity scale in multiple sclerosis patients. Acta Neurol Scand 138:408-416

37. Johns MW (1991) A new method for measuring daytime sleepiness: the Epworth sleepiness scale. Sleep 14(6):540-545

38. Bogdan A, Barnett C, Ali A, Al Qwaifly M, Abraham A, Mannan S, Ng E, Bril V (2019) Chronic stress, depression and personality type in patients with myasthenia gravis. Eur J Neurol 27(1):204-209

39. Nagane Y, Murai H, Imai T, Yamamoto D, Tsuda E, Minami N, Suzuki Y, Kanai T, Uzawa A, Kawaguchi N, Masuda M, Konno S, Suzuki H, Aoki M, Utsugisawa K (2017) Social disadvantages associated with myasthenia gravis and its treatment: a multicentre cross-sectional study. BMJ Open 7:e013278 
40. Dantzer R, O’Connor JC, Freund GG, Johnson RW, Kelley KW (2008) From inflammation to sickness and depression: when the immune system subjugates the brain. Nat Rev Neurosci 9:46-56

41. Liu Y, Tang X (2018) Depressive syndromes in autoimmune disorders of the nervous system: prevalence, etiology, and influence. Front Psychiatry 9:451

42. Kim YK, Na KS, Shin KH, Jung HY, Choi SH, Kim JB (2007) Cytokine imbalance in the pathophysiology of major depressive disorder. Prog Neuropsychopharmacol Biol Psychiatry 31:1044-1053

43. Sukoff Rizzo SJ, Neal SJ, Hughes ZA, Beyna M, RosenzweigLipson S, Moss SJ, Brandon NJ (2012) Evidence for sustained elevation of IL-6 in the CNS as a key contributor of depressivelike phenotypes. Trans Psychiatry 2:e199

44. Uzawa A, Kanai T, Kawaguchi N, Oda F, Himuro K, Kuwabara S (2016) Changes in inflammatory cytokine networks in myasthenia gravis. Sci Rep 6:25886

45. Twork S, Wiesmeth S, Klewer J, Pöhlau D, Kugler J (2010) Quality of life and life circumstances in German myasthenia gravis patients. Health Qual Life Outcomes 8:129

46. Basta IZ, Pekmezović TD, Perić SZ, Kisić-Tepavčević DB, Rakočević-Stojanović VM, Stević ZD, Lavrnić DV (2012) Assessment of health-related quality of life in patients with myasthenia gravis in Belgrade (Serbia). Neurol Sci 33:1375-1381
47. Blum S, Lee D, Gillis D, McEniery DF, Reddel S, McCombe P (2015) Clinical features and impact of myasthenia gravis disease in Australian patients. J Clin Neurosci 22:1164-1169

48. Braz NFT, Rocha NP, Vieira ÉLM, Barbosa IG, Gomez RS, Kakehasi AM, Teixeira AL (2018) Muscle strength and psychiatric symptoms influence health-related quality of life in patients with myasthenia gravis. J Clin Neurosci 50:41-44

49. Jeong A, Min JH, Kang YK, Kim J, Choi M, Seok JM, Kim BJ (2018) Factors associated with quality of life of people with Myasthenia Gravis. PLoS ONE 13:e0206754

50. Alanazy MH, Binabbad RS, Alromaih NI, Almansour RA, Alanazi SN, Alhamdi MF, Alazwary N, Muayqil T (2019) Severity and depression can impact quality of life in patients with myasthenia gravis. Muscle Nerve 61(1):69-73

51. Magni G, Micaglio GF, Lalli R, Bejato L, Candeago MR, Merskey H, Angelini C (1988) Psychiatric disturbances associated with myasthenia gravis. Acta Psychiatr ScandScandinavica 77:443e5

52. Leach LS, Christensen H, Mackinnon AJ, Windsor TD, Butterworth P (2008) Gender differences in depression and anxiety across the adult lifespan: the role of psychosocial mediators. Soc Psychiatry Psychiatr Epidemiol 43:983-998 